\title{
RELATIONS ENTRE LE NOMBRE D'OVULATIONS ET L'ÉTABLISSEMENT D'UNE GESTATION MULTIFCETALE CHEZ LES VACHES DE RACE FRANÇAISE FRISONNE PIE NOIRE
}

\author{
J.-C. MARIANA, D. CHUPIN et P. MAULÉON \\ avec la collaboration technique de Y. DE Foxtaubert \\ Institut national de la Recherche agronomique \\ Laboratoive de Physiologie de la Reproduction 37 - Nouzilly, France
}

L'importance de la connaissance du niveau de superovulation compatible avec l'établissement d'une gestation multifotale se poursuivant jusqu'à l'obtention de naissances gémellaires est primordiale. Cependant peu de précisions ont été apportées sur ce problème, en particulier chez les Bovins.

Chez les Ovins, Robinson (I95I) a analysé les pertes en blastocystes et en embryons en relation avec le nombre d'œufs pondus, par abattage à différents moments de la gestation. Il a conclu que les oufs provenant d'ovulations multiples sont fécondables mais qu'une mortalité embryonnaire très importante se produit avant l'implantation, le milieu utérin étant incapable de supporter un nombre élevé d'embryons.

Chez les Bovins, la connaissance du taux d'ovulation est assurée généralement par palper rectal pour établir une relation, chez ces mêmes vaches, avec le nombre d'embryons (GoRdon et al., Ig62) ou avec le nombre de veaux nés après saillie (HAMMOND, I949). Ce dernier avait estimé, un peu arbitrairement, qu'au-delà de trois ovulations, il ne pouvait pas y avoir gestations multifœtales.

L'imprécision du palper rectal et le fait que celui-ci puisse éventuellement modifier les résultats de gestation nous ont fait préférer l'observation des ovaires par endoscopie (Mariana, I969) pour définir le nombre d'ovulations chez les Bovins et étudier la relation avec l'établissement de la gestation.

\section{MATÉRIEI, ET MÉTHODES}

60 vaches primipares $F . F . P . N$. de l'expérience B IV, c'est-à-dire les animaux traités en janvier-mars et en juillet, ont servi à l'étude de cette relation.

Les vaches reçoivent le même traitement - I 600 UI de PMSG et I 500 UI de HCG - dans les conditions précédemment décrites; un contrôle d'ovulation par la méthode d'endoscopie (Mariana, I969) est effectué dans les trois à six jours qui suivent la saillie. Toutes les vaches 
sont en effet saillies par un taureau toutes les I 2 heures pendant la durée de l'œstrus. Les nonretours en chaleurs, utilisés comme test de gestation, sont détectés régulièrement deux fois par jour à l'aide d'un taureau vasectomisé pendant toute la durée de la gestation.

Dans le cas où un retour en chaleurs est constaté, on attend l'observation d'une deuxième chaleur à un intervalle voisin de la durée normale d'un cycle pour effectuer un deuxième traite. ment identique PMSG + HCG. La saillie, le contrôle du nombre d'ovulations et le contrôle de gestation se succèdent de la même façon qu'après le premier traitement.

\title{
RÉSULTATS
}

Nous avons considéré dans ces résultats : 58 vaches sur les 60 traitées, la fertilité n'étant pas connue sur 2 vaches éliminées trop tôt après la saillie. Vingt-six de ces vaches ont eu un deuxième traitement mais pour la même raison que ci-dessus, 24 données seulement ont pu être ajoutées à celles obtenues après un seul traitement ; la relation nombre d'ovulations - gestation sera donc établie à partir de ces 82 données mises ensemble, aucune différence n'existant entre les deux groupes de résultats.

La distribution du nombre d'ovulations en réponse au traitement PMSG + HCG est la suivante : 40,2 p. Ioo des animaux ont eu o ou I ovulation, 45, I p. Ioo, 2 à 4 ovulations et $\mathrm{I} 4,7 \mathrm{p}$. Ioo plus de 4 ovulations. Cette distribution est très voisine de celle rapportée au chapitre IV. Pour chaque catégorie de classe d'ovulations, les pourcentages de retours entre 20 et 40 jours et de retours tardifs après plus de 80 jours sont donnés dans le tableau I.

\section{TABLEAU I}

\begin{abstract}
Pourcentage de vaches revenant en chaleurs soit $20-40$ jours, soit 80 jours après saillie naturelle ou gestantes à la suite du traitement (82 vaches):
\end{abstract} $P M S G 1600$ UI et HCG 1500 UI

\begin{tabular}{|c|c|c|c|}
\hline \multirow{2}{*}{$\begin{array}{l}\text { P. } 100 \text { de retours } \\
\text { ou gestantes }\end{array}$} & \multicolumn{3}{|c|}{ Nombre d'ovulations } \\
\hline & $0-1$ & $2-3-1$ & $>4$ \\
\hline Retours $20-40$ jours & 36,4 & 32,4 & 16,7 \\
\hline Retours tardifs $>$ & & & \\
\hline 80 jours $\ldots \ldots \ldots$ & 3,1 & 21,6 & 58,3 \\
\hline Animaux gestants. & 60,6 & 45,9 & 25,0 \\
\hline Nombre de données & 33 & 37 & 12 \\
\hline
\end{tabular}

Le taux de gestation le plus élevé est celui de la classe I ovulation. Celui des trop fortes ovulations, c'est-à-dire plus de 4 ovulations, est faible essentiellement à cause des retours en chaleurs, tardifs. Cette classe a, par contre, le plus faible pourcentage de retours après 20-40 jours à la suite du traitement. Dans la classe des 2-3-4 ovulations, on obtient un taux de gestation intermédiaire. Si nous considérons, à l'intérieur de cette classe, les deux sous-classes : 2 ovulations (20 données) et 3-4 ovulations (I7 données), nous constatons que les taux de gestation sont respectivement de $4 \mathrm{I}, 2$ et de 50,I p. Ioo, c'est-à-dire très voisin des 45,9 p. I0o obtenus finalement 
pour l'ensemble du groupe ; mais, ce taux qui est fonction des retours à 20-40 jours et des retours tardifs à plus de 80 , jours est atteint de façon différente pour les deux sous-classes.

Pour le groupe deux ovulations, le taux de retours à 20-40 jours est élevé : 52,9 p. Ioo, et celui des retours tardifs : 5,9 p. I00, bas : résultats comparables à ceux obtenus chez les vaches à o-I ovulation. Par contre, les taux pour la sous-classe 3-4 ovulations sont respectivement de I 5 p. Ioo et 35 p. Ioo, c'est-à-dire qu'ils s'apparentent à ceux de la classe plus de 4 ovulations qui sont caractérisés par un taux de retours tardifs très élevé.

La classe 2-3-4 ovulations est donc bien une classe intermédiaire entre les deux autres du point de vue des relations entre le nombre d'ovulations et l'établissement de la gestation.

\section{DISCUSSION}

Il est toujours arbitraire de découper la distribution des nombres d'ovulations en classes. Nous avons fait ce choix pour la race $F . F . P . N$,, en fonction du pourcentage de retours tardifs liés de façon étroite à l'augmentation du nombre d'ovulations. Aussi, la classe du nombre d'ovulations souhaitable a-t-elle été définie par une limite inférieure liée à la possibilité d'obtenir des naissances gémellaires, ce qui signifie que la limite inférieure est 2 ovulations, et une limite supérieure qui est celle de 4 ovulations à partir de laquelle le taux de retours tardifs est de plus de 50 p. roo. En appliquant cette même méthode aux résultats de Gordon et al. (rg62), la limite supérieure de cette classe a été définie à 5 ovulations. Les résultats de Gordon et al. ainsi analysés offrent une très grande similitude avec ceux que nous présentons : établissement facile d'une gestation chez les animaux superovulés, mais retours tardifs trop fréquents lorsque le nombre d'ovulations dépasse un certain niveau (tabl. 2).

\section{TABLEAU 2}

Relation nombre d'ovulations et obtention de gestations d'après les résultats de Gordon et al. (1962) - 416 vaches - toutes doses de PMSG (entre 800 et 2000 ) UI sans $H C G$

\begin{tabular}{|c|c|c|c|}
\hline \multirow{2}{*}{$\begin{array}{c}\text { P. } 100 \text { de retours } \\
\text { ou gestantes }\end{array}$} & \multicolumn{3}{|c|}{ Nombre d'ovulations } \\
\hline & $0-1$ & $2-3-1_{1}^{\prime}-5$ & $>5$ \\
\hline $\begin{array}{l}\text { Retours avant } \text { six } \\
\text { semaines } \ldots \ldots \ldots \\
\text { Retours tardifs } \ldots \\
\text { Gestantes } \ldots \ldots \ldots\end{array}$ & $\begin{array}{l}29,2 \\
24,3 \\
46,5\end{array}$ & $\begin{array}{l}16,1 \\
20,4 \\
63,5\end{array}$ & $\begin{array}{l}16,7 \\
65,4 \\
17,9\end{array}$ \\
\hline
\end{tabular}

Le taux anormalement élevé de retours tardifs de 24,3 p. Ioo observé chez les vaches de la classe o et I ovulation est imputable vraisemblablement aux conditions 
expérimentales particulières de cette expérience anglaise qui comportait un contrôle de gestation par palper rectal à 6 semaines. Cette technique semble avoir surtout abaissé le pourcentage d'animaux gestants ayant une seule ovulation, la réduction du nombre d'embryons dans la classe 2 à 5 ovulations n'ayant pas supprimé l'existence de la gestation.

Chez les Ovins (RoBInson, I95I), l'incidence du nombre d'ovulations sur le pourcentage de brebis concevant ne s'observe que pour des taux supérieurs à I2 ovulations. Dans cette espèce, la survie des embryons en fonction du nombre d'œufs pondus montre qu'une régulation conduit généralement à l'établissement d'une gestation avec 2 ou 3 embryons quel que soit le nombre d'ovulations.

Chez les Bovins, 76,4 p. Ioo des animaux gestants de la classe 2 à 4 ovulations ont des gestations multiples avec 2 ou 3 embryons après 5 mois. Aucun animal gestant de la classe plus de 4 ovulations n'a de gestation multiple.

Des résultats semblables découlent de l'expérience anglaise (GoRDon et al., I962), bien que $50 \mathrm{p}$. roo seulement des vaches gestantes de la classe $2-3-4-5$ ovulations ont des gestations multifœtales en fin de gestation.

\section{CONCLUSION}

Lorsque le nombre d'ovulations est supérieur à 4 , la gestation s'établit facilement mais disparaît vers le $2^{\mathrm{e}}$ mois de la gestation (TESTART et al., r 969), entraînant un retour en chaleurs tardif et une perte de temps importante.

\section{RÉSSUMÉ}

Un niveau de superovulation compatible avec des gestations multiples a été défini en déterminant par endoscopie, 6 jours après l'œestrus, le nombre des corps jaunes formés après un traitement hormonal de I 600 UI de PMSG et de I 500 UI de HCG chez 58 vaches saillies destinées à concluire leur gestation jusqu'à la mise bas.

Les vaches non gestantes reviennent en chaleurs, soit entre $2 \mathrm{I}$ et 42 jours après la saillie, soit plus de 80 jours après la saillie dans le cas de retours " tardifs" provenant vraisemblablement d'une mortalité embryonnaire. Lorsque le taux d'ovulation est de I ovulation, le taux de gestation final des animaux est surtout déterminé par les 36,4 p. Ioo de retours en chaleurs entre 21 et 42 jours. Les vaches appartenant à la classe des "plus de 4 ovulations " voient l'excellente fertilité de 83,2 p. IOO à $2 \mathrm{I}-42$ jours très diminuée par des retours tardifs chez $58,3 \mathrm{p}$. Ioo des vaches. La réponse 2-3-4 ovulations est finalement la plus favorable parce que $75 \mathrm{p}$. roo des animaux gestants ont des gestations avec des fœtus doubles et triples, cependant le taux de retours " précoces " des animaux de cette classe de réponse ovarienne s'apparente à celui de la classe " I ovulation ", c'est-à-dire qu'il est plus élevé que chez des vaches non traitées et saillies et les retours " tardifs" sans être aussi fréquents que dans la classe "plus de 4 ovulations " existent dans $2 \mathrm{I}, 6 \mathrm{p}$. Ioo des cas.

\section{SUMMARY}

RELATION BETWEEN THE NUMBER OF OVULATIONS AND THE ESTABLISHMENT OF MULTIFGETAL PREGNANCIES IN FRENCH FRIESIAN COWS

The number of ovulations was determined by endoscopy after an I. M. injection of I,6oo I. U. of PMSG four days before the presumed date of cestrus, and an I. V. injection of I,500 I. U. of $\mathrm{HCG}$ at the beginning of the heat period, depending on the number of corpora lutea six days 
after œstrus. The animals used in this experiment were $5^{8}$ French Friesian cows, mated after the superovulation treatment, and carrying their pregnancies to full-term.

The non-pregnant cows came back into heat either between days $2 \mathrm{I}-42$, or more than 80 days after mating in the case of "delayed return, " the latter probably being due to embryonic mortality.

When the ovulation rate was $\mathrm{I}$, the final pregnancy rate was chiefly determined by the 36.4 p. Ioo returning to heat between days $2 \mathrm{I}-42$. The cows which belonged to the more than $4-$ ovulation class had a $2 \mathrm{I}-42$ day non-return rate of $83.2 \mathrm{p}$. Ioo, but $58.3 \mathrm{p}$. Ioo of those cows presented delayed returns. The cows having had 2,3 , or 4 ovulations formed the most propitious population, for $46 \mathrm{p}$. Ioo were pregnant, and $75 \mathrm{p}$. Ioo of those had multifoetal pregnancies (twin or triplet). However, the early return rate of animals of the one-ovulation class was higher than in untreated, mated cows, and 21.6 p. Ioo were late returns (less frequent than in the morethan-four-ovulation class).

\section{RÉFÉRENCES BIBLIOGRAPHIQUES}

Gordon I., Williams G., Edwards S., 1962. The use of serum gonadotrophin PMSG in the induction of twin pregnancy in the cow. J. Agric. Sci. 59, I43-198.

Hammond J. Jr., I949. Induced twin ovulations and multiple pregnancy in cattle. J. Agric. Sci., 39, $222 \cdot 225$.

Mariana J. C., I969. Technique d'examen in vivo des ovaires chez la Vache. Ann. Biol, anim. Bioch. Biophys., 9., 557-559.

Robinson T. J., I95I. The control of fertility in sheep. Part II. The augmentation of fertility by gonadotrophin treatment of ewe in the normal breeding season. J. Agric. Sci., 41, 6-63.

Testart J., Bosc M. J., du Mesnil du Buisson F, i968. Étude de la survie embryonnaire après induction de superovulation chez la Vache. VI ${ }^{\mathrm{e}}$ Congr. Int. Reprod. anim. Ins. artif., 1, 479-482. 